\title{
Biografische Umgangsformen mit sexuellem Missbrauch durch katholische Geistliche Ergebnisse einer qualitativen Interviewstudie mit Betroffenen
}

\author{
Sandra Fernau
}

\section{Einleitende Bemerkungen}

Die Problematik des sexuellen Missbrauchs an Minderjährigen durch katholische Geistliche erhielt mit dem Bekanntwerden zahlreicher Vorfälle in den Vereinigten Staaten und verschiedenen Ländern Europas seit den 1990er Jahren weltweit größere öffentliche Aufmerksamkeit. Nichtsdestotrotz lässt sich für diesen Bereich national wie auch international eine Forschungslücke feststellen: Im Gegensatz zur Vielzahl an empirischen Untersuchungen zu sexueller Gewalt in familiären Umgebungen (zum Überblick: z. B. Finkelhor, 1994; Lampe, 2002), existieren nur wenige Studien zu sexuellem Missbrauch in kirchlichen Kontexten. Der überwiegende Teil dieser Arbeiten stammt aus den Vereinigten Staaten, in denen ab den 1990er Jahren eine wissenschaftliche Auseinandersetzung mit der Problematik stattfand; seit der öffentlichen Missbrauchsdebatte 2010 entstehen vereinzelt auch Studien im deutschsprachigen Raum.

Die mehrheitlich quantitativen Untersuchungen befassen sich aus einer vornehmlich psychologischen Perspektive vor allem mit den klerikalen Tätern und möglichen Ursachen ihres sexuell devianten Verhaltens. Erforscht werden etwa deren Persönlichkeitsmerkmale und sexuelle Störungen, wie beispielsweise Pädophilie oder Ephebophilie (z. B. Doyle, 2003; Haywood, Kravitz, Grossman, Wasyliw \& Hardy, 1996; Haywood, Kravitz, Wasyliw, Goldberg \& Cavanaugh, 1996; Laaser, 1991; Leygraf, König, Kröber \& Pfäfflin, 2012; Plante, 2003; Plante, Manuel \& Bryant, 1996). Darüber hinaus findet in den vergangenen Jahren vermehrt eine Analyse von psychosozialen Folgen der Missbrauchserfahrungen für die Betroffenen statt. Der Fokus liegt hierbei zumeist auf psychotraumatologischen Fragestellungen (z. B. Bottoms, Nielsen, Murray \& Filipas, 2003; Carr et al., 2010; Lueger-Schuster, 2012); teilweise werden auch Auswir- 
kungen der Vorfälle auf religiös-spirituelle Einstellungen bzw. die spirituelle Dimension der Traumatisierung von Betroffenen in den Blick genommen (z. B. Benkert \& Doyle, 2009; Doyle, 2006, 2009). Als bedeutende und umfassende quantitative Untersuchungen gelten zwei groß angelegte, vom John Jay College of Criminal Justice im Auftrag der United States Conference of Catholic Bishops (USCCB) durchgeführte Studien: zum einen der „Nature and Scope Report“ über die Prävalenzraten sexueller Übergriffe durch katholische Priester und Diakone im Zeitraum von 1950 bis 2002; zum anderen der „Causes and Context Report“, der eine soziohistorische und psychologische Analyse der Vorkommnisse innerkirchlichen sexuellen Missbrauchs vornimmt und Erklärungsansätze für mögliche Ursachen und Hintergründe der Sexualdelikte liefert (John Jay College, 2004; Terry et al., 2011). ${ }^{1}$

An einem qualitativen Forschungsparadigma orientierte viktimologische Studien mit einer sozialwissenschaftlichen Perspektive gibt es hingegen bislang kaum. Allerdings ermöglicht gerade ein qualitativer Zugang zum Forschungsfeld durch die eingehende Untersuchung individueller Reaktionsmuster, spezifischer Dynamiken und Auswirkungen vertiefende Erkenntnisse über Besonderheiten von sexuellen Missbrauchserfahrungen in katholischen Kontexten. Eine soziologische Ausrichtung wiederum eröffnet den Blick auf institutionell-strukturelle Hintergründe, Bedingungen und Charakteristika religiöser Sozialisation sowie deren Einfluss auf die Betroffenen und ihre Auseinandersetzung mit der sexuellen Gewalt. Hierdurch können vertiefende Erkenntnisse über biografische Umgangsformen mit innerkirchlichem Missbrauch, aber auch über Wirkweisen von religiöser Sozialisation und Religiosität geliefert werden.

Die am Kriminologischen Forschungsinstitut Niedersachsen (KFN) im Rahmen des Projekts „Sexueller Missbrauch durch katholische Geistliche“ durchgeführte qualitative Interviewstudie mit Betroffenen greift diese Desiderate auf. Ihr zentrales Erkenntnisinteresse besteht darin, biografische Deutungsmuster und Verarbeitungsversuche innerkirchlicher Viktimisierungserfahrungen zu rekonstruieren sowie diese vor dem Hintergrund einer religiösen Sozialisation und ihrer Einwirkung auf die Betroffenen und deren Umgang mit dem Erlebten zu analysieren. Im vorliegenden Beitrag werden wesentliche Ergebnisse der Untersuchung vorgestellt. Die Vorge-

1 Zur ausführlicheren Darstellung des nationalen und internationalen Forschungsstands, siehe Kapitel 2 dieses Sammelbands (Fernau, Treskow \& Stiller, 2014) sowie die Literaturexpertise des Deutschen Jugendinstituts (Bundschuh, 2010). 
hensweise der Darstellung lässt sich so skizzieren: Zur näheren Bestimmung des Einflusses und der Rolle von Glauben und religiöser Praxis werden zuerst einige grundlegende religionssoziologische Annahmen aufgegriffen. Sodann erfolgt eine kurze Beschreibung des der Untersuchung zugrunde liegenden Forschungsdesigns und der Zusammensetzung des Samples der Interviewstudie. Im Fokus des Beitrags steht die Rekonstruktion von kontrastierenden Deutungs- und Verarbeitungsmustern der sexuellen Missbrauchserfahrungen. Anhand ausgewählter Interviewsequenzen soll ein typisierender Überblick zu den unterschiedlichen biografischen Umgangsformen der Betroffenen mit dem Erlebten geliefert werden, wobei der häufig anzutreffende Rückgriff auf religiöse Interpretationsschemata einen Schwerpunkt der Betrachtung bildet.

\section{Zum Einfluss von Religion und religiöser Sozialisation auf das Individuum}

Ausgehend von der Annahme, dass die katholische Erziehung und Sozialisation die Persönlichkeitsentwicklung prägt und somit auch einen Einfluss auf den Umgang mit dem sexuellen Missbrauch hat, folgen zunächst einige Überlegungen zu Religion und religiöser Sozialisation. Im Mittelpunkt stehen dabei die Bestimmung zentraler Merkmale und Funktionen von Religion bzw. Religiosität sowie die Einwirkung von Glauben und religiöser Praxis auf das Individuum.

\section{II.1 Zum Religionsbegriff}

In Anlehnung an klassische und moderne religionssoziologische Definitionen (z. B. Durkheim, 1981; Knoblauch, 1999; Parsons, 1964; Pickel, 2011; Pollack, 1995, 2001; Stark \& Glock, 1968) lässt sich Religion bestimmen als ein System von Symbolen, Glaubensvorstellungen und Praktiken, die sich auf übernatürliche und als heilig betrachtete Wesen, Dinge und Kräfte einer jenseitigen Welt beziehen. Den Kern der Religion bildet ihr letzter, nicht mehr einzuholender Sinn, das über das Bestehende hinausgehende und somit ihr Transzendenzbezug. In institutionalisierter Form gibt sie Glaubenssätze und handlungsleitende Vorschriften, ethische Richtlinien und moralische Ideale für die jeweilige Glaubensgemeinschaft vor. 
In dieser Begriffsbestimmung sind zentrale Merkmale benannt, die aus der Vielfalt soziologischer Religionsdefinitionen herausgearbeitet werden können und sich in unterschiedlicher Ausprägung auch in der gelebten Religion, also der (persönlichen) Religiosität wiederfinden. Allgemein wird auf vier Bestimmungselemente verwiesen: Erstens sind dies die individuellen Überzeugungen der Gläubigen, ihre Glaubensvorstellungen, welche sich auf höhere Mächte allgemein und Gott im Speziellen richten. Hinzu kommen die (sozialen) Praktiken, die sich als charakteristische Handlungen vor allem in religiösen Ritualen und Zeremonien (wie bspw. der Taufe, der Trauung und dem Gottesdienst) vollziehen. Da die beiden zuerst genannten Charakteristika von Religion meist in Zusammenhang mit Regeln des jeweiligen religiösen Kollektivs stehen, verweisen sie drittens auf eine moralische Gemeinschaft, die sich in der Einhaltung der gleichen Normen und Verpflichtungen ausdrückt. Häufig hat diese Gemeinschaft eine institutionelle Ausprägung: Sie äußert sich in Form der Kirche als gesellschaftliche Organisation, die Religion (und Religiosität) institutionalisiert und handlungsleitende Vorgaben für die Gläubigen formuliert (Pickel, 2011; Pollack, 1995, 2001).

\section{II.2 Zum nachhaltigen Einfluss religiöser Sozialisation}

Im Rahmen einer im traditionellen Sinne stark religiös beeinflussten Sozialisation ist die Lebensführung und Weltdeutung eng mit Normen, Glaubensvorstellungen und -praktiken verbunden, die von der Kirche vorgegeben werden. Diese erhalten sowohl im alltäglichen Familienleben als auch durch das Eingebundensein in die kirchliche Gemeinde und die hiermit einhergehende rituelle religiöse Teilhabe eine besondere Bedeutung auf perzeptiver, kognitiver und interaktiver Ebene: Sie formen das Erleben, Deuten und alltagspraktische Handeln der Gläubigen. Die auf diese Weise im Zuge familiärer Erziehung und religiöser Sozialisation verinnerlichten Glaubensüberzeugungen und Wertorientierungen erweisen sich als nachhaltig prägend (Knoblauch, 1999; Lorenzer, 1981; Pickel, 2011). Sie können zwar im Laufe der Zeit nachlassen und unterliegen in der Stärke ihrer Ausprägung zumeist lebenszyklischen Schwankungen, aber dennoch bilden die frühzeitig internalisierten Grundhaltungen die Basis für spätere Einstellungsmuster gegenüber Religion und Kirche. Ganz in diesem Sinne belegen vielfältige empirische Forschungsergebnisse die große Bedeutung der Sozialisation für Religiosität und die Bindung an die 
Kirche im weiteren Verlauf des Lebens (z. B. Breuer, 2012; Pickel, 2011; Pollack, 2003; Stolz, 2008).

Das von Lorenzer (1981) formulierte Konzept von Religion bzw. Religiosität bietet einen theoretischen Erklärungsansatz für diesen tiefgreifenden Einfluss religiöser Sozialisation. Seinem aus der Psychoanalyse entwickelten sozialisationstheoretischen Konzept folgend, ist es vor allem die über die sprachliche Glaubensbotschaft hinausreichende, sich auf der Wirkungsebene der Bilder entfaltende religiöse Symbolik, durch welche Religion frühzeitig und nachhaltig auf die Persönlichkeitsentwicklung einwirkt. Als präsentatives Symbolsystem beeinflusst sie die Persönlichkeitsbildung auf der Stufe sinnlich-symbolischer Interaktionsformen. Auf diesem sinnlich-bildhaften Organisationsniveau entsteht laut Lorenzer im primären Sozialisationsprozess das individuelle Bewusstsein: Es wird eine „erste Ich-Struktur“ (Lorenzer, 1981, S. 162) gebildet, die „Identität der Person begründet" (S. 214) und durch die Verinnerlichung kultureller Traditionen und Bräuche gleichzeitig ,der Zusammenhang mit dem Kollektiv gestiftet“ (S. 214). Dabei ermöglichen unter anderem das Gottesdienstritual mit seiner szenischen Gestalt und musikalischen Praxis sowie auch die Architektur des Kirchenraums in ihrer räumlich-bildlichen Erlebnisqualität Glaubenserfahrungen auf der Stufe sinnlich-symbolischer Interaktionsformen. In besonderem Maße trifft jene Wirkweise von Religion auf den Katholizismus mit seiner stark ritual- und mythenträchtigen Glaubenspraxis sowie den oftmals opulent gestalteten Kirchenbauten und liturgischen Gewändern der Geistlichen zu (Busch, 2007; Lorenzer, 1981).

\section{II.3 Zu (biografischen) Funktionen von Religion}

Wichtige soziologische und sozialpsychologische Funktionen von Religion, die in zahlreichen Publikationen Erwähnung finden, lassen sich wie folgt zusammenfassen: Wie bereits erwähnt, werden im Zuge religiöser Sozialisation und der Eingliederung in ein religiöses Kollektiv zentrale Bestandteile des Glaubens-, Werte- und Symbolsystems der jeweiligen Glaubensgemeinschaft internalisiert. Insofern kommt der Religion eine identitätsstiftende Funktion zu: Sie leistet einen wesentlichen Beitrag zur Konstitution der individuellen Identität und deren Erhaltung im Wandel. Über die Verinnerlichung der Glaubensüberzeugungen werden dabei zum einen Möglichkeiten für den Ausdruck und die Artikulation eigener Emotionen und Wünsche geliefert, zum anderen Bewertungsmaßstäbe für das 
eigene Handeln und das der Anderen bereitgestellt (Knoblauch, 1999; Parsons, 1964; Pickel, 2011).

Darüber hinaus wird der Religion eine integrative, gemeinschaftsstiftende Funktion zugeschrieben: Mittels der Bildung eines religiösen Kollektivs durch geteilte Glaubens- und Wertvorstellungen sowie rituellsymbolische Gemeinschaftserlebnisse entsteht ein überaus starkes $\mathrm{Zu}$ sammengehörigkeitsgefühl ihrer Anhängerinnen und Anhänger, bis hin zur Empfindung eines „,ungeschiedenen Einsseins“ (Busch, 2007, S. 150), eines „ozeanischen Gefühls“ (Freud, 1930, S. 197). Aus der sozialen Kohäsion resultiert nicht nur eine Stärkung der Bereitschaft zur aktiven Beteiligung am kirchlichen Leben, sondern ebenso eine Überführung der egoistischen Wünsche und Ängste des Einzelnen in Gruppenziele. Hierdurch werden für das Gemeinwesen potenziell bedrohliche Situationen entschärft (Knoblauch, 1999; Milanesi, 1976; Pickel, 2011).

In Bezug auf den Umgang mit verschiedenen biografischen Erfahrungen bieten religiöse Konstrukte durch ihre ordnende und sinnstiftende Interpretation der Welterfahrung der bzw. dem Einzelnen eine Orientierungs-, Deutungs- und Bewältigungshilfe. Dies gilt insbesondere für problematische Lebenslagen, konflikt- und krisenhafte Erfahrungen. Mit ihrer sinngebenden Bewertung findet eine Entdramatisierung von Widersprüchen und Konflikten, eine Entschärfung bedrohlicher oder emotional belastender Situationen statt (Knoblauch, 1999; Parsons, 1964; Storch, 2000). Die hierdurch ausgelösten ermutigenden oder tröstenden Gedanken führen zu einer Angst- und Stressreduktion. Ferner trägt eine sinnverleihende Bewertung des eigenen Handelns sowie des Lebens allgemein zur Steigerung des Selbstwertgefühls bei und vermittelt positive Gefühle wie Hoffnung und Freude. Auf diese Weise fungieren religiöse Überzeugungen innerhalb der intrapsychischen Emotions- und Verhaltensregulation als Befriedigungs- und Bewältigungsstrategien (Grom, 2007; Haslbeck, 2007).

Ein Spezifikum der religiösen Bewältigung biografischer Konflikt- und Krisenerfahrungen hängt mit dem der Religion inhärenten Transzendenzbezug zusammen. Dieser ermöglicht eine Transzendierung von Erwartungserfüllungen: Die in der Gegenwart (bzw. allgemein im Diesseits) unerfüllten Hoffnungen und Erwartungen werden in eine nicht überprüfbare, transzendentale Zukunft verlagert. Durch das religiöse Versprechen einer jenseitigen Erlösung erfolgt so eine Kompensation des als unverdient empfundenen Leids oder der als ungerecht bewerteten Lebensereignisse (Knoblauch, 1999; Pickel, 2011). In Zusammenhang mit dieser Form religiöser Problemlösung steht auch die von Pollack $(1995,2003)$ in Anleh- 
nung an Luhmann $(1977,1987)$ beschriebene Funktion der Kontingenzbewältigung. Hiermit ist die Lösung existenzieller Fragen und Probleme, hervorgerufen vor allem durch Krisenerfahrungen (wie bspw. die Konfrontation mit Tod und Krankheit) durch die Verlagerung der Verantwortung ins Jenseits gemeint. Indem der Glaube an die Stelle einer rationalen Erklärungsstrategie tritt, findet eine Transzendierung des Problems statt, die der Entlastung des Selbst dient (Pickel, 2011; Pollack, 1995, 2003; Storch, 2000).

Abschließend soll noch kurz auf zwei grundsätzliche Funktionen eingegangen werden, die Religion allgemein im biografischen Kontext erfüllt: Zum einen erweist sich Religion in ihrer rituellen, kirchlich institutionalisierten Form insbesondere bei lebensgeschichtlichen Übergängen als bedeutsam. Hier übernimmt sie in erster Linie eine ,lebensgeschichtlichordnende Funktion" (Wohlrab-Sahr, 1995, S. 9) mittels der durch sie zur Verfügung gestellten Passageriten, ${ }^{2}$ die Übergänge in verschiedene Lebensphasen begleiten, rahmen und zu bearbeiten helfen (Storch, 2000; Wohlrab-Sahr, 1995). Zum anderen kommt der Religion eine ,reflexive Funktion" (Wohlrab-Sahr, 1995, S. 9) im Rahmen der Auseinandersetzung mit der eigenen Biografie zu. Diese setzt ebenfalls vor allem bei lebensgeschichtlichen Übergängen ein, die potenziell krisenhaft sein können und daher biografisch gestaltet und reflektiert werden müssen. Religion dient hier als Motiv und Mittel der Selbstthematisierung und -beobachtung: Während Glaubensvorstellungen (wie bspw. die Hoffnung auf Erlösung oder die Angst vor Strafe) eine biografische Reflexion anregen und lenken, werden innerhalb des institutionellen Kontexts bestimmte Formen der Selbstthematisierung (z. B. die Beichte) bereitgestellt.

\section{Methode und Sample}

Die Interviewstudie orientierte sich in ihrem Ablauf an wesentlichen Prinzipien der Grounded Theory (Przyborski \& Wohlrab-Sahr, 2010; Strauss, 1998; Strauss \& Corbin, 1996). In drei Erhebungsphasen wurden im Zeitraum von Herbst 2011 bis Frühling 2013 insgesamt 15 biografisch angelegte, teilnarrative Leitfadeninterviews (Helfferich, 2009; Kruse, 2014) mit erwachsenen weiblichen und männlichen Betroffenen sexuellen Miss-

2 Dazu zählen die Taufe, Kommunion, Firmung oder Konfirmation, die kirchliche Trauung und Beerdigung. 
brauchs durch katholische Geistliche geführt. Unter die Bezeichnung „katholische Geistliche" fallen hier katholische Priester (auch Kapläne bzw. Vikare), Diakone und männliche Ordensangehörige, die im Bereich der Deutschen Bischofskonferenz tätig waren bzw. sind. Es handelt sich also ausschließlich um männliche Täter. Ausgewertet wurde das erhobene Material ebenfalls in Anlehnung an zentrale Arbeitsschritte der Grounded Theory. Die Kontaktierung der Gesprächspartnerinnen und -partner fand auf drei Wegen statt: mittels einer Kontaktaufnahme durch die Betroffenen selbst, über die Weiterleitung eines Anschreibens des KFN durch die Missbrauchsbeauftragten eines Bistums an ihnen bekannte, potenzielle Teilnehmerinnen und Teilnehmer sowie mit Hilfe eines öffentlichen Aufrufs des KFN in verschiedenen Medien. Bei der Auswahl der Interviewten wurde eine heterogene Zusammensetzung des Samples unter anderem hinsichtlich des Geschlechts und Alters sowie des Missbrauchskontextes angestrebt, um ein möglichst breites Spektrum an Erfahrungen, Deutungsund Verarbeitungsmustern erschließen zu können. ${ }^{3}$

Tabelle 1. Samplezusammensetzung der qualitativen Interviewstudie $(N=15)$

\begin{tabular}{lrrr}
\hline & $\begin{array}{r}\text { Alter zum Zeitpunkt } \\
\text { des Interviews }\end{array}$ & $\begin{array}{r}\text { Alter zum Zeitpunkt } \\
\text { des Missbrauchs }\end{array}$ & $\begin{array}{r}\text { Dauer des } \\
\text { Missbrauchs }\end{array}$ \\
\hline Spanne & $37-71$ Jahre & 4-17 Jahre & 0.5-9 Jahre \\
Durchschnitt & 58 Jahre & 11 Jahre & 3.5 Jahre \\
\hline
\end{tabular}

Von den insgesamt 15 teilnarrativen, leitfadengestützten Interviews wurden drei mit Frauen und zwölf mit Männern geführt. Die Altersspanne der Befragten zum Zeitpunkt des Interviews reicht von 37 bis 71 Jahren und beträgt im Durchschnitt 58 Jahre (siehe Tabelle 1). Die sexuellen Missbrauchserfahrungen der Betroffenen liegen also bereits mehrere Jahrzehnte zurück. Alle der von ihnen berichteten, in den Einzelheiten sehr unterschiedlichen Missbrauchshandlungen fanden mit Körperkontakt statt; erfolgten diese zunächst ohne Berührungen, so trat der Körperkontakt im Laufe der Zeit hinzu. Es wurden sexuelle Aktivitäten mit, vor und auch an den Betroffenen geschildert. Zum Zeitpunkt des Beginns der sexuellen

3 Eine ausführliche Darstellung der Kontaktierungswege, des Samplingverfahrens sowie der Erhebungs- und Auswertungsmethoden der qualitativen Interviewstudie enthält Kapitel 3 dieses Sammelbands (Fernau \& Hellmann, 2014). 
Übergriffe waren die Befragten zwischen 4 und 17 Jahren alt; durchschnittlich entwickelte sich die Missbrauchsbeziehung ab dem elften Lebensjahr. Der sexuelle Missbrauch ging in den allermeisten Fällen über mehrere Jahre und erstreckte sich mit Blick auf das gesamte Sample über einen Zeitraum zwischen ca. einem halben Jahr und neun Jahren.

Die Kontexte, in denen die sexuellen Übergriffe stattfanden, sind heterogen: Es wurden Personen mit Missbrauchserfahrungen in geschlossenen Institutionen wie Heimen und Internaten ebenso wie Betroffene, die in allgemein kirchengemeindlichen Zusammenhängen - beispielsweise im Rahmen ihrer Messdienerschaft - sexuelle Gewalt erlebten, interviewt. Darüber hinaus finden sich im Sample mehrere Betroffene, die in privaten Kontexten von katholischen Geistlichen missbraucht wurden - zum Beispiel bei Besuchen im Pfarrhaus in ihrer Freizeit, während Nachhilfeunterrichtsstunden und auf katholischen Jugendfreizeiten. Grundsätzlich besteht bei allen Befragten ein großer Altersunterschied zwischen ihnen und dem Täter, der für das Forschungsfeld innerkirchlichen sexuellen Missbrauchs charakteristisch sein dürfte. In der vorliegenden Interviewstudie beträgt die Altersdifferenz stets mehrere Jahrzehnte, wobei die beschuldigten Geistlichen mindestens 30 Jahre alt (im Falle von Kaplänen), vorwiegend jedoch über 50 Jahre alt waren (Pfarrer).

\section{Biografische Umgangsformen mit den sexuellen Missbrauchs- erfahrungen}

Bevor eine Analyse der rekonstruierten Umgangsformen mit den sexuellen Übergriffen erfolgt, soll kurz auf zwei allgemeine Aspekte eingegangen werden, die in den meisten biografischen Erzählungen eine wichtige Rolle spielen und für das Verständnis der Deutungs- und Verarbeitungsmuster bedeutend sind.

Zum einen handelt es sich dabei um die Problematik der Aufdeckung des Erlebten, die auch in der einschlägigen Forschung zu sexuellem Missbrauch als charakteristisch für den Umgang mit solchen Viktimisierungserfahrungen gilt. So belegen verschiedene empirische Untersuchungen, dass ein großer Teil derartiger Vorfälle nicht oder erst mit erheblicher zeitlicher Verzögerung aufgedeckt wird (z. B. Bange, 2007; London, Bruck, Cesi \& Shuman, 2005; Mosser, 2009; siehe auch Wollinger, Dinkelborg \& Baier, 2014; Kap. 7 in diesem Band). Für die Befragten der vorliegenden Interviewstudie trifft dies ebenfalls zu: Die meisten von ihnen brachten ihre Missbrauchserfahrungen erst viele Jahre nach den Vorfällen zur Spra- 
che; mehr als die Hälfte der Betroffenen hat jahrzehntelang über die sexuellen Übergriffe geschwiegen. Erst mit der breiten öffentlichen Aufdeckung von Missbrauchsvorfällen in staatlichen und kirchlichen Einrichtungen im Jahre 2010 offenbarten sie ihre Erlebnisse, allerdings zumeist lediglich sehr wenigen ausgewählten Personen. Bis heute haben manche von ihnen die Vorfälle ihrem näheren familiären und sozialen Umfeld nicht mitgeteilt. Wie sich in mehreren Deutungsmustern der Interviewten zeigt, sind mit der Aufdeckungsproblematik über missbrauchsspezifische (und somit vom jeweiligen Kontext unabhängige) Dynamiken hinaus auch sich aus dem religiösen Setting ergebende Besonderheiten verknüpft.

Zum anderen weisen viele Narrative in unterschiedlichen Zusammenhängen Bezüge zu Elementen katholischen Glaubens auf. Diese in den verschiedenen Schilderungen aufscheinenden religiösen Prägungen lassen sich unter anderem mittels der im vorherigen Abschnitt beschriebenen Wirkweise religiöser Sozialisation erklären. In der Erziehung und Sozialisation der interviewten Betroffenen fand eine enge Verbindung der Lebensführung und Weltauslegung mit religiösen Ordnungssystemen und Vorlagen statt. Im alltäglichen Leben in ihren zumeist sehr religiösen Herkunftsfamilien, die stark in die örtliche Kirchengemeinde eingebunden waren, sowie in den streng nach religiösen Vorschriften reglementierten Internaten und Heimen spielten diese eine zentrale Rolle. Demnach erhielten katholische Glaubensvorstellungen eine besondere Bedeutung und formten das Erleben, Deuten und alltagspraktische Handeln der Interviewten. Dieser frühkindliche Einfluss religiöser Sozialisation ist so nachhaltig, dass er sich auf unterschiedliche Weise bis heute in den meisten biografischen Erzählungen allgemein sowie in den geschilderten Reaktionsmustern im Umgang mit dem sexuellen Missbrauch zeigt. Es ist also in vielen Narrativen eine mehr oder weniger deutlich ausgeprägte Orientierung an katholischen Glaubensvorstellungen auszumachen, die sich im Rückgriff auf religiöse Semantiken zur Weltauslegung wie zur Selbstinterpretation und Deutung lebensgeschichtlicher (Krisen-)Ereignisse manifestiert. Dieses teils individualisierte Festhalten an katholischen Glaubenszusammenhängen wird auch daran sichtbar, dass Gebete (wie bspw. Tischgebete, Fürbitten, Andachten) für mehrere Interviewte einen festen Bestandteil in ihrem alltäglichen Leben darstellen. Darüber hinaus spielt mit der Einbindung in die örtlichen kirchengemeindlichen Strukturen die Teilhabe an der institutionell ritualisierten Glaubenspraxis eine wichtige Rolle für einen Teil der Befragten. Einige sind bis heute Mitglieder der katholischen Kirche, manche von ihnen nehmen aktiv an kirchlichen Angeboten teil, besuchen regelmäßig den sonntäglichen Gottesdienst und engagieren sich eh- 
renamtlich in der Gemeinde. Alle Betroffenen, die verheiratet sind, ließen sich katholisch trauen und ihre Kinder katholisch taufen, die (wie sie selbst) größtenteils ebenfalls als Messdienerinnen bzw. Messdiener tätig waren oder sind.

Mit Blick auf die sexuellen Missbrauchserfahrungen lassen sich insgesamt fünf Umgangsformen der interviewten Betroffenen identifizieren, in denen sich größtenteils der Einfluss von Glauben und religiöser Praxis andeutet. $^{4}$ Im Folgenden sollen diese Deutungsmuster und die mit ihnen zusammenhängenden Verarbeitungsversuche der Missbrauchserfahrungen konkretisiert werden. Sie können klassifiziert werden als:

- eine Angst vor Stigmatisierung durch das familiäre und soziale Umfeld sowie eine oftmals hiermit verbundene Angst vor dem antizipierten Verlust der Zugehörigkeit zur Familie und kirchlichen Gemeinde,

- Scham- und Schuldgefühle, die in eine Selbstanklage der Betroffenen münden,

- eine Bagatellisierung der Missbrauchsvorfälle, die zur Ablehnung bzw. Verleugnung des eigenen Betroffenenstatus führt,

- eine religiöse, sinnverleihende Umbewertung des Geschehenen,

- eine Übernahme der Deutung des Täters, durch welche eine Legitimation des sexuellen Missbrauchs stattfindet.

\section{IV.1 Die Angst vor Stigmatisierung und dem Verlust der Zugehörigkeit zur Familie und kirchlichen Gemeinde}

Das subjektive Erleben eines großen Teils der interviewten Betroffenen ist damals wie heute von Ängsten hinsichtlich möglicher Folgen der Aufdeckung ihrer Erlebnisse geprägt: Es herrscht eine tief verankerte Angst vor ablehnenden Reaktionen, Schuldzuweisungen und Stigmatisierungen durch das Umfeld und einem hiermit verbundenen Verlust von Zugehörigkeiten zur Familie und kirchlichen Gemeinde.

4 Hierbei handelt es sich um eine typisierende Rekonstruktion, die aufgrund der verschiedentlichen Relationen zwischen den einzelnen Umgangsformen in Teilen auch anders hätte ausfallen können. Eine detailliertere und stärker auf den Einzelfall bezogene Rekonstruktion ausgewählter religiöser Deutungsmuster des sexuellen Missbrauchs durch katholische Geistliche findet sich bei Fernau (2013). 
Die beiden nachstehenden Äußerungen zweier Betroffener illustrieren die Antizipation negativer Zuschreibungen durch das familiäre und soziale Umfeld bei einer Aufdeckung ihrer Missbrauchserfahrungen besonders deutlich ${ }^{5}$ :

\begin{abstract}
„Vielleicht habe ich gedacht, wenn ich das jetzt sage, dann falle ich irgendwie aus der doch vorhandenen Beziehung bei meinen Eltern raus, dann bin ich markiert, dann bin ich als schwarzes Schaf markiert. Dann bist du vielleicht, ja und zwar in negativer Weise bin ich dann markiert. Und da ist etwas Schlimmes dann mit dir passiert, was von deinen Eltern vielleicht auch als ganz abscheulich angesehen wird, und vielleicht können die dir auch gar nicht verzeihen oder da ist irgendwas Irreparables, so etwas ganz Negatives passiert, und ich wollte vielleicht auch den Rest an Zuwendung und den Rest an Liebe [...] nicht verlieren.“
\end{abstract}

Während es sich bei dieser Aussage eines heute 71-jährigen Mannes um eine retrospektive Deutung seiner subjektiven Situation in der Zeit des Missbrauchs und den Jahren danach handelt, beziehen sich die folgenden Ausführungen einer 37-jährigen weiblichen Betroffenen auf ihre momentan empfundene Lage:

\begin{abstract}
„Also, da würde ich mich nirgends mehr hin trauen, da würde es überall, auch in der Gemeinde heißen ,Guck mal, die kommt da, das Missbrauchsopfer' und so. Also, das wär mir total unangenehm. Und vor allen Dingen geht's mir eigentlich auch um meine Kinder [...] Also, das würde ich jetzt meinen Kinder nie zumuten wollen. [...] Dass dann irgendjemand da mit dem Finger auf meine Kinder zeigt oder auf uns, also nee. [...] Da kann es noch so oft heißen, wir können eigentlich nix dafür, aber ich glaub, dass die Leute trotzdem auf einen zeigen würden. Man, man hat dann einfach so 'nen Makel anheften.“
\end{abstract}

Neben dem Verschweigen der Missbrauchsvorfälle aus Angst, als betroffene Person mit einem Stigma gebrandmarkt zu werden und die Schuld

5 Die im Folgenden angeführten Zitate aus den Interviews entsprechen weitestgehend dem tatsächlichen Sprachgebrauch der Befragten; sie sind lediglich zugunsten der Lesbarkeit angepasst worden. Damit ist gemeint, dass Pausen, inhaltsleere Füllwörter (wie bspw. ,äh“), nonverbale Gesprächssignale, Betonungen und dergleichen hier aus der Transkription entfernt wurden. Von der Autorin vorgenommene Kürzungen innerhalb der jeweiligen Schilderungen sind mit [...] markiert. 
hierfür zugeschrieben zu bekommen, klingt in beiden Schilderungen auch die Befürchtung an, durch eine Aufdeckung der Erlebnisse Zugehörigkeiten zu verlieren. Insbesondere im ersten Zitat zeigt sich die Angst vor dem antizipierten Verlust wichtiger positiver Beziehungselemente zur Familie. Diese wurde in der damaligen Situation des Betroffenen dadurch verstärkt, dass der Täter als Priester und Gemeindeoberhaupt eine besondere Machtund Autoritätsposition in der örtlichen Gemeinschaft innehatte. Er galt als moralische Instanz und Wächter der Tugend, mehr noch: als von Gott Berufener und als Vermittlungsinstanz zwischen den gläubigen Laiinnen bzw. Laien und Gott (Doyle, 2003, 2006; Plante, 1996). Der mit jener hervorgehobenen Stellung verbundene Einfluss des Priesters reichte auch in die familiären Strukturen des Interviewten hinein; er erscheint auf seine in das Gemeindeleben sehr integrierte Herkunftsfamilie gar so groß, dass der Interviewte bei einer Offenbarung der sexuellen Übergriffe befürchtete, dass seine Eltern ihm nicht ,verzeihen“ können, er also selbst die Schuld für die Missbrauchsvorfälle angelastet bekäme. Im zweiten Zitat deutet sich erneut die Antizipation negativer Zuschreibungen und Schuldzuweisungen bei einer Aufdeckung des Erlebten an. Hier richten sich die bei der Interviewpartnerin bis heute bestehenden Ängste jedoch stärker auf eine befürchtete Stigmatisierung durch das soziale bzw. kirchliche Umfeld und eine hiermit verbundene Ausgrenzung aus der Gemeinschaft.

Vergleichbare Ängste vor ablehnenden Reaktionen des Umfelds und einer möglichen Ausgrenzung arbeiten auch Keupp, Straus, Mosser, Gmür und Hackenschmiedt (2013) in ihrer qualitativen Interviewstudie zu Gewaltvorkommnissen im Internat der Benediktinerabtei Ettal als eine Erklärung für das Schweigen von Betroffenen über die Missbrauchsvorfälle heraus. Dabei geht es allerdings eher um die von Betroffenen vorgenommene retrospektive Deutung ihrer damaligen Lage, währenddessen die hier anklingenden Befürchtungen teils bis in die Gegenwart fortbestehen. Derart begründete Ängste vor Stigmatisierungen sowie dem Verlust bedeutsamer sozialer und institutioneller Bindungen sind insbesondere für diejenigen Interviewten kennzeichnend, denen eine bis heute bestehende Teilhabe am zumeist dörflichen Gemeindeleben gemeinsam ist. Sie beziehen sich vor allem auf einen möglichen Ausschluss aus der örtlichen Kirchengemeinde und einem damit einhergehenden Verlust wichtiger sozialer Kontakte. Eine offene Thematisierung und Aufarbeitung der Viktimisierungserfahrungen innerhalb der Glaubensgemeinschaft bleibt hier in der Regel aus: Zwar fand im Zuge der öffentlichen Missbrauchsdebatte eine Meldung der erlebten sexuellen Gewalt bei den zuständigen Missbrauchsbeauftragten des jeweiligen Bistums statt. Allerdings war die Mitteilung in 
den allermeisten dieser Fälle mit dem Wunsch verknüpft, namentlich nicht genannt zu werden, und die Wahrung der Anonymität erhielt einen sehr großen Stellenwert.

Eine Verarbeitung der Missbrauchserfahrungen im Zuge einer Aufdeckung und aktiven Hilfesuche im sozialen und kirchlichen Umfeld wird bei jenen Betroffenen gerade durch ihre bis heute bestehende starke emotionale Verbundenheit und Loyalität zur örtlichen religiösen Gemeinschaft erschwert: So lassen die primär auf kollektivistischen Wertorientierungen basierenden Sozialisationserfahrungen den möglichen Verlust ihrer kirchengemeindlichen Bindungen in der Folge einer Offenbarung ihrer Erlebnisse als besonders bedrohlich erscheinen. Eine Aufdeckung wird vor diesem Hintergrund mit einer Entscheidung über das Fortbestehen der eigenen sozialen Integration in Verbindung gebracht. Sie stellt sich in den Augen der Betroffenen als schuldbehaftete Auflösung wichtiger Bindungen dar, als Verrat am Täter und an der Gemeinde. Anstelle der unter anderem von Summit (1983) und Crisma, Bascelli, Paci und Romito (2004) für innerfamiliären sexuellen Missbrauch beschriebenen charakteristischen Angst vor dem Zerfall der Familie, tritt hier also die Angst vor dem Zerfall der Gemeindezugehörigkeiten, die Angst vor dem Verlust des Gemeinschaftsrückhalts in den Vordergrund des subjektiven Erlebens der Betroffenen. In beiden Fällen handelt es sich um die Angst vor dem $\mathrm{Zu}$ sammenbruch elementarer (Beziehungs-)Strukturen, die Halt und Sicherheit geben. ${ }^{6}$

\section{IV.2 Die Selbstanklage von Betroffenen}

Wie sich in den beiden obigen Zitaten bereits andeutet, gehen die beschriebenen Ängste vor Stigmatisierung und dem Verlust von Zugehörigkeiten häufig mit starken Scham- und Schuldgefühlen seitens der Be-

6 Eine Ausnahme von den in diesem Abschnitt beschriebenen Erlebnis- und Deutungsmustern bildet der Umgang von zwei weiteren Studienteilnehmern: Sie wurden nach dem Bekanntwerden der zahlreichen Missbrauchsvorfälle in kirchlichen Bereichen öffentlich aktiv und nahmen an Treffen von Betroffenenverbänden und politischen Untersuchungsgremien teil. Einer von ihnen berichtete in Fernseh- und Zeitungsinterviews über seine traumatischen Erlebnisse und setzt sich in verschiedenen institutionellen Kontexten für die Aufarbeitung und Prävention sexuellen Missbrauchs an Schutzbefohlenen ein. Beide Interviewpartner zeigen sich allerdings als sehr distanziert von religiösen Überzeugungen und Praktiken, haben sich also weitestgehend von kirchlichen Zusammenhängen gelöst. 
troffenen einher. Diese führen dann meist zu einer Selbstanklage, in der sich die Betroffenen die Verantwortung für die Missbrauchsvorfälle selbst zuschreiben.

Ganz in diesem Sinne erläutert die oben zitierte Interviewpartnerin ihre Gedanken und Gefühle gegenüber Betroffenen von sexuellem Missbrauch allgemein und somit indirekt auch in Bezug auf sich selbst:

\begin{abstract}
„Man ist ja doch immer irgendwie so 'nen bisschen, innerlich überkommt es einen ja dann, dass man denkt, na ja, das Opfer wird vielleicht aufreißerisch rumgerannt sein oder hat es vielleicht ja selber gewollt oder ist ja selber schuld. Also, aus der Rolle komm ich eben auch nicht raus.“
\end{abstract}

Vergleicht man diese Aussage mit ihren obigen Ausführungen, wird auBerdem deutlich, dass die Befragte die von ihr befürchteten negativen Fremdzuschreibungen selbst gegen sich wendet. Ihre Angst vor Stigmatisierung wird also durch Selbstvorwürfe mitbedingt und zugleich verschärft.

Die Selbstbezichtigung hängt mit einer bis heute andauernden verzerrten Wahrnehmung der damaligen Situation wie der eigenen Rolle zusammen: So bringt die Interviewte mit der Mutmaßung, dass Opfer sexueller Übergriffe ,vielleicht aufreißerisch rumgerannt“ seien und „es vielleicht ja selber gewollt" haben, indirekt nicht nur die Annahme einer Freiwilligkeit und Komplizenschaft zum Ausdruck, mehr noch: Sie erweckt implizit Anschein, den Geistlichen mit dem eigenen Auftreten betört und so erst zu seinen Handlungen verlockt zu haben. Dementsprechend manifestiert sich in ihrem Deutungsmuster eine Umkehrung der tatsächlichen Rollen in der Missbrauchssituation: die Interviewte macht sich als Betroffene selbst zur Täterin.

In der nachfolgenden Schilderung eines zum Zeitpunkt des Interviews 65 Jahre alten Betroffenen wird ebenfalls eine Selbstbezichtigung deutlich. Der auch gegenwärtig in der örtlichen Kirchengemeinde aktive Mann hat seine Missbrauchserfahrungen bis heute weder dem familiären noch dem sozialen oder kirchlichen Umfeld offenbart. Sein subjektives Erleben ist damals wie aktuell von Befürchtungen hinsichtlich möglicher Folgen der Aufdeckung seiner Erlebnisse geprägt: einer Angst vor Stigmatisierung durch das Umfeld sowie dem hiermit verbundenen antizipierten Verlust der Zugehörigkeit zur dörflichen und kirchlichen Gemeinschaft. Wie sich im Interview an einigen Stellen zeigt, besteht auch hier ein Zusammenhang zwischen der Angst vor negativen Fremdzuschreibungen und 
Selbstvorwürfen. Die hieraus resultierende Selbstanklage des Betroffenen erhält durch die Einbettung in eine religiöse Deutung allerdings eine noch weiter reichende Konnotation:

\begin{abstract}
„Also, und wenn man, wenn so 'ne Gemeinde nachher überhaupt gar kein Gemeindeleben mehr hat, warum, weil so 'ne ganze Generation weggebrochen ist, wie in \{A-Dorf\} da, dann muss ja was passiert sein, ne? Und und immer mit diesem Kainsmal zu leben, das sagen die ja im Dorf dann weiter, wird erzählt, so ' $n$ Dorf ist ja klein. So. Und und immer so zu leben, du hast mit dem Pastor was gehabt oder so. [...] Das bleibt im Dorf. Das ist doch, das ist doch so. Das das geht nicht weg. [...] Ja, weil jemand, der so was erlebt hat in so 'nem Dorf und es und es wird, bleibt nicht anonym... Deswegen bin ich auch da nicht mit groß groß rumgelaufen oder so, ne? Sondern ich hab gesagt, das behältst du jetzt für dich.“
\end{abstract}

Indem der Interviewte darauf verweist, dass er (bei einer Aufdeckung seiner Erlebnisse) als Betroffener ein für alle im Ort sichtbares „Kainsmal“ tragen würde, bringt er eine schuldbehaftete Angst vor Stigmatisierung zum Ausdruck, welcher er eine religiöse, von Gott verliehene Bedeutung zuschreibt. So ist die von ihm verwendete Bezeichnung dem Alten Testament der Bibel entlehnt (1. Mose 4,1-16) und stammt aus der Geschichte der Brüder Kain und Abel. In dieser erschlägt Kain aus Neid seinen Bruder Abel wegen dessen vermeintlich bevorzugter Stellung zu Gott bei einem Gottesopfer. Als Strafe für den Brudermord wird er von Gott verflucht und erhält ein Zeichen, das ihn für sein weiteres Leben brandmarkt. Es hat zweierlei Bedeutung: Das Kainsmal ist einerseits das Erkennungszeichen des aus der Gemeinschaft ausgestoßenen Mörders und somit ein Schandmal. Andererseits stellt es ein Schutzzeichen dar, das Kain als nur von Gott zu Richtenden kennzeichnet und auf diese Weise vor einem gewaltsamen Tode bewahrt. Es handelt sich also im biblischen Sinne um eine gerechte Bestrafung eines Straftäters durch Gott.

In Bezug auf die (Selbst-)Wahrnehmung des Befragten bedeutet dies, dass er seines Erachtens aufgrund der Missbrauchserfahrungen ein Zeichen der Schuld und Schande trägt, das ihn verrät und brandmarkt. Jedoch ist das Kainsmal kein von Menschen allgemein oder Personen des sozialen Umfelds zugeschriebenes Stigma, sondern ein Zeichen von Gott. Deswegen ist auch kein Entkommen aus der Rolle des Gebrandmarkten möglich: Der Betroffene muss ,immer mit diesem Kainsmal [...] leben“. Geleitet wird die Selbstanklage des Interviewten also von der Vorstellung, durch den erlebten sexuellen Missbrauch eine Schuld vor Gott auf sich geladen 
zu haben, weshalb sich die hierfür zu tragende Stigmatisierung als eine gerechte Bestrafung erweist. In dem hier skizzierten religiösen Deutungsmuster wird demnach wiederum eine Umkehrung der tatsächlichen Rollen in der Missbrauchssituation deutlich: Der Interviewte macht sich als Betroffener zum Täter bzw. Sünder. Im Zuge der Selbstinterpretation im Rahmen einer katholischen Semantik weist er sich selbst die Schuld für die sexuellen Gewalterfahrungen zu.

\section{IV.3 Die Bagatellisierung der Missbrauchsvorfälle}

Neben dem soeben benannten Zusammenhang zwischen der Angst vor Stigmatisierung und einer Selbstanklage, lässt sich ein weiterer ausmachen. So ist letztere nicht selten mit einer Bagatellisierung oder tendenziellen Verleugnung der sexuellen Missbrauchserfahrungen verbunden bzw. hat einen ähnlich gearteten Deutungsursprung. All jene Verarbeitungsversuche resultieren zumeist aus der Deutung der Betroffenen, sich gegen den Missbrauch nicht angemessen zur Wehr gesetzt zu haben - sowohl physisch als auch durch eine Vermeidung des Kontakts mit dem Geistlichen. Auf diese Weise unterliegen viele der Betroffenen noch Jahrzehnte nach den Übergriffen der Suggestion von Freiwilligkeit und Komplizenschaft. Jene verzerrte Wahrnehmung geht dann oftmals mit einer Selbstanklage oder aber mit einer Bagatellisierung bzw. tendenziellen Verleugnung der erlebten Gewalt einher. Insofern hängt die Selbstbezichtigung als Wendung gegen die eigene Person sowie die Bagatellisierung der Erlebnisse als verharmlosende Reinterpretation mit einer bis heute andauernden Fehleinschätzung der damaligen Situation und der eigenen Rolle in dieser zusammen.

Jene Betrachtungsweise kommt auch in dem zuletzt angeführten Zitat zum Ausdruck. In der euphemistischen Aussage ,du hast mit dem Pastor was gehabt" zeigt sich, dass der Betroffene bis heute den Missbrauch als einvernehmliche sexuelle Beziehung umdeutet; mehr noch: Sie erweckt sogar implizit den Anschein eines eigenen sexuellen Fehlverhaltens, so als ob die sexuellen Handlungen von ihm ausgegangen seien. Ein anderer Interviewter bezeichnet seinen Kontakt zum Täter mehrfach als „Beziehung“, womit ebenfalls eine Freiwilligkeit und Komplizenschaft suggeriert wird.

Auch die nachstehende Äußerung eines Betroffenen weist in diese Richtung: 
„Als das mit den Missbrauchsfällen hoch kam, da habe ich es dann auch gesagt, dass ich also auch verführt worden bin, ja? Und, dass es 'nen Pastor... Aber ich habe das nicht so hoch gehängt. Ich hänge das auch nicht so hoch wie wie Menschen, die wirklich missbraucht worden sind, ja?“

In der hier getroffenen Unterscheidung zwischen „verführt“ und „,missbraucht" worden zu sein klingt erneut die Einschätzung an, sich gegen die Übergriffe nicht ausreichend zur Wehr gesetzt zu haben, gegebenenfalls selbst in der Situation mit ambivalenten Gefühlen und einer sexuellen Erregung konfrontiert gewesen zu sein. So verweist zum Beispiel Bange (2007) darauf, dass für einige sexuell missbrauchte Jungen die Einordnung der Situation durch das Auftreten eigener erregender Empfindungen zusätzlich erschwert wird. Hierdurch entsteht noch stärker der Eindruck, den Missbrauch selbst auch gewollt zu haben und aktiv am Geschehen beteiligt gewesen zu sein. Hinzu kommt möglicherweise der Wunsch einer Aufrechterhaltung von positiven Beziehungselementen zum Geistlichen, welcher dazu beiträgt, das Geschehene zu verharmlosen.

Oftmals werden die bei Betroffenen auftretenden Schuldgefühle durch Neutralisierungstechniken des Täters, die dem Betroffenen suggerieren, die sexuellen Handlungen selbst auch gewollt zu haben, verschärft. Derartige Manipulationen, die für den Bereich innerfamiliären Missbrauchs als charakteristisch gelten (Bange, 2007; Mosser, 2009) und zur Verzerrung von Wahrnehmungsmustern führen, sind demnach auch Missbrauchskonstellationen im kirchlichen Bereich inhärent. Hier zeigt sich die Wirkmächtigkeit der vom Täter zur Etablierung der Missbrauchsbeziehung angewandten Strategien, die häufig auch längere Zeit nach der Beendigung des sexuellen Missbrauchs die Gefühle und die (Selbst-)Wahrnehmung von Betroffenen beeinflussen. Im Falle der später dargelegten religiösen Neutralisierungstechniken, die dem Betroffenen eine religiöse Legitimation oder gar Notwendigkeit der sexuellen Handlungen suggerieren, erweisen sich die Auswirkungen auf das Selbstbild der Betroffenen als umso schwerwiegender.

Darüber hinaus dienen manchen männlichen Betroffenen des Samples eine Selbstanklage wie auch eine Bagatellisierung als Schutz gegen die durch den Kontrollverlust ausgelösten Gefühle der Hilflosigkeit und Ohnmacht. Indem sich die Betroffenen selbst die Schuld an den Vorfällen geben oder diese herunterspielen, können sie sich die Illusion erhalten, in der damaligen Situation zumindest eine gewisse Kontrolle gehabt zu haben. Die Vorstellung, dass sie es hätten besser machen können bzw. dass es gar nicht so schlimm war, erscheint mit ihrem Selbstbild als Mann eher 
vereinbar, als sich der Tatsache des vollkommenen Ausgeliefertseins zu stellen (Bange, 2007; Herman, 1994). Deshalb qualifiziert ihr subjektives Deutungsschema die Vorfälle auf eine Weise, die eine Einschätzung der eigenen Person als Betroffener nicht zulässt.

Die folgenden Auszüge aus einem Interview mit einem zum damaligen Zeitpunkt 66-jährigen Mann deuten ebenfalls auf ein solches Interpretationsmuster hin:

„Das Unangenehme, weswegen ich ja nun auch hier sitze, das hatte ich eigentlich völlig vergessen, sehr schnell, hat mich auch nicht belastet, ja? In meiner Kindheit oder bei dem bei diesen Erlebnissen, aber irgendwie hab ich gedacht, da stimmt was nicht, ja? Und deswegen wollte ich da aus dem Internat wieder weg, ja?"“

Gleich zu Beginn des Interviews stellt der Betroffene mit der zitierten Aussage klar, dass ihn die sexuellen Übergriffe selbst zum Zeitpunkt des Missbrauchs emotional nicht belastet haben. Im weiteren Verlauf seiner biografischen Erzählung macht er an mehreren Stellen deutlich, dass er auch in der Folgezeit in keiner Weise unter den Erfahrungen gelitten hat. Exemplarisch sei hierzu die nachstehende Sequenz angeführt:

\begin{abstract}
„Also, ich ich hab eben keine Probleme mit der Bewältigung gehabt wie viele, was man ja jetzt hört und was publik geworden ist. Und dann habe ich das aber auch wieder vergessen und erst als hier vor ein, zwei Jahren das losging in der Öffentlichkeit, da da ist mir das wieder eingefallen, ja? Aber ich gehöre zu den glücklichen Menschen, will ich mal so sagen, die keinen Schaden genommen haben. [...] Also, es ist nicht so, dass ich jetzt eben äh über meine Probleme sprechen will, ich hab keine Probleme damit gehabt, ja? [...] Es hat sich in meiner Entwicklung nicht irgendwie äh hat mich beeinträchtigt oder so. Ist also nicht der Fall.“
\end{abstract}

Im hier zitierten Interviewauszug betont der Befragte wiederholt vehement, dass er sowohl unmittelbar nach dem Missbrauch als auch gegenwärtig, nachdem ihm im Zuge der breiten medialen Aufdeckung von Missbrauchsvorfällen die eigene Betroffenheit erneut vor Augen geführt wurde, „keine Probleme“ mit der Bewältigung des Erlebten hatte. Obwohl es sich hierbei offenkundig um eine jahrzehntelange Verdrängung der traumatischen Erfahrungen (als (Selbst-)Schutz) handelt, schreibt der Interviewte diesen keinerlei Bedeutung zu. Auf diese Weise grenzt er sich 
explizit von allen Betroffenen ab, die öffentlich über bis heute bestehende psychosoziale Folgen und Belastungsreaktionen berichten und teilweise auch erst mit der öffentlichen Debatte ihre eigene Betroffenheit realisierten. Gleichzeitig demonstriert er im Rekurs auf ein traditionelles Bild von Männlichkeit Härte und Stärke. Seine Verarbeitungsstrategie der Viktimisierungserfahrungen besteht also in einer Negation des eigenen Opferstatus bzw. jeglicher hiermit verbundener Konsequenzen. ${ }^{7}$ Derartige Verleugnungen und Bagatellisierungen werden in einschlägigen Veröffentlichungen als gängige Bewältigungsstrategien von insbesondere männlichen Betroffenen sexuellen Missbrauchs genannt (z. B. Finkelhor, 1979; Mosser, 2009; Wetzels, 1997).

\section{IV.4 Die religiöse, sinnverleihende Umbewertung des Missbrauchs- geschehens}

Ein weiteres Reaktionsmuster, das sich bei manchen Befragten in je unterschiedlich stark ausgeprägter Form zeigt, besteht in einer religiös konnotierten, positiven Umbewertung der Geschehnisse. Indem sie den sexuellen Missbrauchserfahrungen im Rückgriff auf religiöse Glaubensvorstellungen nachträglich einen Sinn verleihen, findet eine Minderung der durch sie ausgelösten emotionalen Belastungen statt: Gefühle des Schmerzes, der Wut und Trauer werden mittels der sinnstiftenden Deutung kompensiert. Auf diese Weise dienen die im Rahmen der katholischen Erziehung und Sozialisation verinnerlichten religiösen Überzeugungen innerhalb der intrapsychischen Emotions- und Verhaltensregulation der Interviewten als Bewältigungsressource ihrer traumatischen Erfahrungen.

Zwei Äußerungen eines heute 41-jährigen Betroffenen illustrieren diesen Rückgriff auf religiöse Interpretationsvorlagen zur Verarbeitung des sexuellen Missbrauchs besonders augenfällig:

7 An dieser Stelle ist darauf hinzuweisen, dass sexueller Missbrauch nicht immer massive psychische Belastungen nach sich ziehen muss. Die Häufigkeit und Vehemenz der Negierung jeglicher negativer Auswirkungen sowie der Umstand, dass die Vorfälle Jahrzehnte lang nicht erinnert wurden, legt jedoch die Vermutung nahe, dass es sich hierbei um eine (dem Interviewten in dieser Form nicht bewusste) Bagatellisierung der Vorfälle handelt. 
„Und die Schwierigkeiten, die kommen, so gut wie möglich anzunehmen und überhaupt dann das Göttliche in jedem anderen Menschen zu sehen und überhaupt in allen Situationen des Lebens das Göttliche zu sehen und keinen $\mathrm{Zu}$ fall, sondern einen göttlichen Lehrer, der mir etwas sagen möchte. Und Demut ist dann, ja, wenn ich etwas auf den Deckel bekomme, dass ich das dann annehme von Gott, einfach, weil ich die Widmung habe, alles als von Gott zu betrachten."

Über die Einbettung der Missbrauchsvorfälle in eine religiöse Welterfahrung und die Annahme eines göttlichen Determinismus findet hier eine sinnstiftende, positive Umdeutung des Erlebten statt. Dabei kommt es zu einer zweifachen Umbewertung der sexuellen Gewalterfahrungen: Mit dem Bekenntnis ,,in allen Situationen des Lebens das Göttliche zu sehen und keinen Zufall, sondern einen göttlichen Lehrer, der mir etwas sagen möchte" werden diese zunächst in eine Prüfung Gottes verwandelt, durch die der Interviewte eine Möglichkeit erhält, sich Gott als würdig zu erweisen. Die Aussage ,weil ich die Widmung habe, alles als von Gott zu betrachten" markiert schließlich die Transformation des sexuellen Missbrauchs in ein Geschenk Gottes. So lässt sich unter der auffälligen Formulierung einer „Widmung“ die besondere, durch Gott verliehene Gabe des Interviewten verstehen, die jahrelangen erlebten sexuellen Übergriffe als Teil eines für ihn vorgesehenen göttlichen Plans zu erkennen. Weil er den Willen Gottes demütig befolgt, wird sein Gehorsam mit einer göttlichen Zuwendung belohnt, sodass er aus den leidvollen Erfahrungen etwas Positives ziehen kann. Ganz in diesem Sinne erläutert der Befragte im weiteren Verlauf des Interviews, dass jene Sichtweise sein Leben „sehr bereichert" habe und er insofern trotz des sexuellen Missbrauchs „doch sehr beschenkt wurde“".

Diese religiös geprägte positive Umbewertung der Geschehnisse ermöglicht dem Interviewten eine Kompensation der durch den sexuellen Missbrauch hervorgerufenen Gefühle von Hilflosigkeit und Verzweiflung sowie des erlebten Schmerzes. Sämtliche Symptome und Belastungsreaktionen, die bei dem Betroffenen auch langfristig nach den Viktimisierungserfahrungen auftraten, erhalten einen höheren Sinn; sie dienten in seiner Interpretation der eigenen Persönlichkeitsentwicklung.

Darüber hinaus erfahren die sexuellen Übergriffe des Priesters im Zuge dieser Umdeutung eine Legitimation: Sie erscheinen nicht länger als Tat eines verantwortlichen Menschen, sondern vielmehr als Teil eines göttlichen Plans. In diesem Sinne handelt nicht nur der Befragte selbst, sondern eben auch der Täter nach Gottes Willen. Zugleich sind aufgrund seiner re- 
ligiösen Überzeugung, dass alles, was im diesseitigen Leben passiert, Gott gegeben ist, alle Geschehnisse wie auch deren Beurteilung in Gottes Hand gelegt. Deshalb wäre es für den Interviewten auch nicht infrage gekommen, eine Strafanzeige gegen den Priester zu stellen; vielmehr wird in seiner Deutung Gott ein gerechtes Urteil für den Täter sprechen.

Dementsprechend antwortet er auf die Frage der Interviewerin „Sie haben auch nicht darüber nachgedacht oder Ihre Mutter, eine Strafanzeige zu stellen?" folgendermaßen:

„Ich hab nicht drüber nachgedacht, nee. Ja, ich hab immer die Auffassung vertreten dann, ja? Oder ich hab immer versucht dann zu sehen dann, wie es mir doch dann trotzdem geholfen hat dann, ja? Der Mensch zu werden, der ich heute bin dann, ja? Das hat mich sicherlich dann sehr viel sensibler gemacht und sehr viel aufmerksamer gemacht dann, was das Leiden von anderen Menschen anbelangt dann, und das ist sehr gefragt heute dann, ja? Das heißt, ich kümmere mich sehr um um viele Menschen dann. Also, die auch Rat suchen dann und arbeite sehr viel ehrenamtlich. Aber vielleicht wäre das alles nicht so möglich gewesen dann, ja? Ohne, dass man durch einen so leidvollen Prozess gegangen wäre. So das ist damals schon dann so meine Auffassung gewesen. Und das war der Grund dann, warum ich gesagt hab nee, das ist nicht meine Aufgabe hier ein Urteil zu fällen, das muss der Herrgott dann machen dann, ja? Nach dem Tod möglicherweise dann, ja? Dann wird er die Konsequenzen sicherlich ernten."

Der Beginn des Zitats veranschaulicht erneut die oben bereits beschriebene positive Umbewertung des Missbrauchsgeschehens, die in eine Transformation der sexuellen Gewalterfahrungen in ein göttliches Geschenk mündet. In der Selbstdeutung des Interviewten hat der sexuelle Missbrauch ihm nicht nur trotz bzw. aufgrund der schmerzvollen Erfahrungen „geholfen [...] der Mensch zu werden“, der er heute ist. Zusätzlich wird er von Gott mit einer besonderen Zuwendung bedacht und bekommt als Belohnung für das erfahrene Leid außergewöhnliche Fähigkeiten von ihm verliehen, die in der heutigen Gesellschaft ,sehr gefragt“ seien: So haben ihn die Erfahrungen in seiner Wahrnehmung gegenüber anderen Menschen ,sehr viel sensibler [...] und sehr viel aufmerksamer gemacht“.

In den Ausführungen zeigt sich außerdem die in den religionssoziologischen Überlegungen zu Beginn des Artikels bereits skizzierte Verlagerung von Erwartungserfüllungen in eine transzendente Sphäre, die im Rückgriff auf religiöse Glaubensvorstellungen vor sich geht und dem Individuum eine Entlastung bietet. Indem der Befragte seinen Strafwunsch gegenüber 
dem Täter in einen jenseitigen Bereich verlagert, findet ein Umgehen der weltlichen Gerichtsbarkeit statt; alles, was im Diesseits passiert, löst sich im transzendenten Rahmen einer religiösen Weltanschauung auf. Den Umschlagpunkt hierfür bildet der Tod: Nach diesem wird der Geistliche in den Augen des Interviewpartners eine gerechte Strafe von Gott für seine Tat erhalten. Somit findet durch den Glauben an eine göttliche Bestrafung des Täters im Jenseits eine Kompensation des erlebten Leids und Schmerzes statt. Zugleich werden Wutgefühle und aggressive Impulse gegenüber dem Täter mittels der Transzendierung ausgeglichen. Die auf diese Weise stattfindende Entlastung bedeutet allerdings auch, dass all jene schmerzhaften und wutbesetzten Affekte im diesseitigen Leben nicht zugelassen werden und daher zu keiner Handlung führen. Vielmehr tröstet die Vorstellung von einer jenseitig hergestellten Gerechtigkeit durch Gott über derartige Empfindungen hinweg.

\section{IV.5 Religiöse Neutralisierungstechniken von Geistlichen und deren Übernahme durch Betroffene}

In Zusammenhang mit der Etablierung von Missbrauchsbeziehungen spielen die Strategien, mittels derer es dem Täter gelingt, die Betroffenen gefügig zu machen und die Geheimhaltung der sexuellen Handlungen sicherzustellen, eine zentrale Rolle. Zugleich beeinflussen sie während und auch nach der Beendigung des sexuellen Missbrauchs die Gefühle, die (Selbst-)Wahrnehmung und das Verhalten der Betroffenen. Eine Verwicklung in Missbrauchsbeziehungen kann auf sehr unterschiedliche Art und Weise geschehen. Das Spektrum reicht von einer langsamen, subtilen Gewöhnung an eine Atmosphäre der Übergriffigkeit bis hin zu drastischen Drohungen und der Anwendung körperlicher Gewalt, wobei auf letztere in der Regel aufgrund des sozial- und entwicklungsbedingten Machtgefälles zwischen Täter und Betroffenen verzichtet werden kann (Bange, 2007; Wetzels, 1997).

Eine gängige, in der Fachliteratur häufig benannte Täterstrategie besteht im Aufbau einer Vertrauensbeziehung zum Betroffenen. Durch eine besondere emotionale und soziale Zuwendung soll dem Kind das Gefühl vermittelt werden, etwas Besonderes zu sein. Dabei werden vor allem solche Mädchen und Jungen ausgewählt, die aufgrund ihrer emotionalen, familiären oder sozialen Situation als besonders vulnerabel erscheinen (Bange, 2007; Bullens, 1995). Es findet also in der Regel ein zielgerichte- 
tes Vorbereiten des sexuellen Missbrauchs statt, das auch als ,grooming process" (Berliner \& Conte, 1990, S. 30) bezeichnet wird.

In kirchlichen Kontexten kommen spezifische Täterstrategien hinzu, die als religiöse Manipulation der Betroffenen einzustufen sind. Es handelt sich dabei um einie Instrumentalisierung religiöser Glaubensvorstellungen zur Begründung und Rechtfertigung der sexuellen Übergriffe. Als Beispiele für solche religiös geprägten Neutralisierungstechniken lassen sich folgende nennen: Zunächst berichten mehrere Interviewte von sexuellen Handlungen im Rahmen der Beichte. Meist wurden diese unter dem Vorwand der Bestrafung für sündige Gedanken bzw. ein untugendhaftes Verhalten, vereinzelt auch als Beichtersatz (im Sinne einer Entbindung vom Mitteilen unsittlicher Phantasien) vollzogen. Ein weiterer Betroffener schildert, dass der Geistliche vorgab, ihn im Zuge der angewandten Sexualpraktiken selig zu sprechen. Darüber hinaus wurden die Missbrauchshandlungen in Bußrituale eingebunden. Indem der Täter den Betroffenen dazu anhielt, nach dem Sexualakt mit ihm zu beten und damit gemeinsam für die begangene Sünde Buße zu tun, suggerierte er diesem, dass die Handlungen einvernehmlich stattgefunden haben. Auf diese Weise entsteht beim Betroffenen der Eindruck, diese selbst auch gewollt und dadurch eine Schuld auf sich geladen zu haben.

Einer Befragten, bei welcher der sexuelle Missbrauch im Alter von vier Jahren begann, wurde vom Priester über Jahre hinweg bescheinigt, er müsse ihr mit seinen Handlungen den Teufel austreiben. Durch die Einbettung der sexuellen Übergriffe in Exorzismen verlieh er seinen Taten nicht nur eine Notwendigkeit, ja Dringlichkeit, sondern erzeugte bei der vermeintlich von Dämonen befallenen Interviewten zugleich tiefe Schamund Schuldgefühle, verbunden mit der Empfindung, verdorben und von Grund auf böse zu sein.

Der nachstehende Interviewauszug, in denen die Betroffene die Missbrauchshandlungen schildert, veranschaulicht die perfide Vorgehensweise des Täters: 
„Und er hat mir dann erklärt, ja im Prinzip, ich hätte schlechtes Blut. Meine Oma mit 17 schon schwanger geworden. Meine Mutter ein uneheliches Kind, nämlich mich. Und das käme alles vom Teufel. Und den müsste er mir jetzt austreiben. [...] Später musste ich dann das Höschen ausziehen. Und er hat mich manipuliert. Er hat mich so lange manipuliert bis ich als Kind sexuelle Gefühle bekam. Um mir dann zu erzählen ,Diese Gefühle, das sind die Gefühle, vor denen du dich hüten musst. Die darfst du nicht haben. Und jedes Mal, wenn du die hast, lieferst du sozusagen einen Nagel zum Kreuz des Heilands.' Und ja. Na, ich hab natürlich versprochen, ich werd werd die nie wieder haben. Und ja. ,Das werden wir dann beim nächsten Mal rauskriegen.' Und ja. Er hat mich jedes Mal so lange manipuliert, bis ich die Gefühle wieder hatte, ne? ,Siehst du, der Teufel ist immer noch in dir und wir müssen noch mal.' Und ja. ,Und das wird erst zu Ende sein, wenn du diese Gefühle nicht mehr kriegst'.“

All jene skizzierten Tatstrategien sind dadurch gekennzeichnet, dass die Geistlichen die durch sie vermittelten katholischen Glaubensvorstellungen zur Etablierung einer Missbrauchsbeziehung instrumentalisieren. Diese religiöse Manipulation verstärkt die bei den Betroffenen ausgelösten Gefühle von Hilflosigkeit und Ohnmacht, vor allem aber auch das Empfinden von einer Legitimität und Normalität, teils gar Notwendigkeit der Handlungen des Täters. Auf diese Weise wird in den allermeisten Fällen eine Geheimhaltung der Taten gewährleistet.

Darüber hinaus zeigt sich in den Narrativen einzelner Betroffener bis heute der Einfluss der religiösen Neutralisierungstechniken auf ihre Selbstdeutung und Wahrnehmung des Geschehens. So findet sich auch Jahrzehnte nach den Missbrauchsvorfällen in manchen Darstellungen (indirekt) eine Reproduktion der religiös begründeten Rechtfertigungsstrategien der Geistlichen. Eine wichtige Rolle spielen hierbei die im Zuge der religiösen Sozialisation vermittelten Glaubensinhalte, die - auch bei einer im rationalen Bewusstsein weitgehend erfolgten Emanzipation hiervon bis heute eine prägende Wirkung entfalten.

Der nachstehende Interviewauszug veranschaulicht, wie diese Verhaftung in katholischen Glaubensvorstellungen eine vollständige Abkehr von den vom Täter herangezogenen Deutungsweisen erschwert. Es handelt sich dabei um einen heute 66-jährigen Mann, der in einem katholischen Internat mehrmals von einem Priester sexuell missbraucht wurde. Den Tathergang schildert er zunächst wie folgt: 
„Also, es war so, wenn wir abends um, ich glaube um halb zehn sind wir da ins Bett, und das war ja ein großer Schlafsaal, und dann ist irgendwann vielleicht nach 'ner Stunde oder so, ja? Sind die dann immer rein gekommen die Padres und haben geguckt, ob wir schlafen. Man hat ja als Kinder hat man ja auch rumgealbert und so. Und dann wurde halt immer jemand rausgeholt, ja? Und eines Tages wurde ich halt auch mal rausgeholt. Und dann musste ich mitkommen in sein Zimmer und [...] sehe den also noch. Der saß dann also hier in so 'nem dicken Sessel, ja? Und ich stand vor ihm, und dann hat er die Schlafanzughose mir mir runter gezogen, ja? Und hat gesagt ,Hast du eine Sünde begangen?' ,Nein'. ,Hast du onaniert?' [...] Und dann hat er da rumgespielt, ja?“

Seine daran anschließende Deutung lautet:

„Und und irgendwie, ja ich war war mir dessen gar nicht bewusst, ja? Was der will eben, ,Hast du gesündigt', ja? ,Hast du onaniert, hast du 'ne Sünde begangen?', ja? Und vielleicht hab ich damals schon 'nen gewissen Drang nach Sünde gehabt."

Wie sich den Schilderungen entnehmen lässt, fanden die sexuellen Übergriffe hier unter dem Deckmantel der Überprüfung sündigen Verhaltens statt. Der Betroffene, der im weiteren Verlauf des Interviews diesen Vorwand klar benennt, greift nichtsdestotrotz an dieser Stelle das vom Täter verwendete Rechtfertigungsmuster fast im Wortlaut auf. So schreibt er sich selbst einen ,gewissen Drang nach Sünde“ zu, obgleich er bekennt, sich damals (im Alter von elf Jahren) „dessen gar nicht bewusst“ gewesen zu sein, was der Priester mit seiner Frage nach einer möglichen Selbstbefriedigung meinte. Indem er sich dennoch selbst rückblickend als Sünder bezichtigt, erscheint sein eigenes damaliges (und letztlich auch heutiges) Verhalten und nicht mehr das des Priesters als verwerflich. Die Tat wird auf diese Weise zu einer notwendigen Handlung, mehr noch: zu einer gerechten Strafe für seine vermeintlichen kindlichen Vergehen. Hierdurch erfährt sie in der Deutung des Interviewten eine Legitimation.

Dabei tragen insbesondere die vom Befragten verinnerlichten religiösen Glaubensmuster, die sich im Rekurs auf katholische Vorstellungen zeigen, zu einer verzerrten Wahrnehmung seiner Betroffenheit sowie der damaligen Situation bei. Mit dem Rückgriff auf die Sündenkategorie wendet er auch gegenwärtig eine Religionskategorie auf sich selbst an, interpretiert sich also noch immer im Rahmen der Semantik, welche die katholische Kirche und vor allen Dingen das Beichtritual anbietet. Dies macht seine nachhaltige religiöse Prägung auch in Bezug auf die katholische Sexual- 
moral deutlich: Onanie oder auch Geschlechtsverkehr, der außerhalb der Ehe und nicht zum Zwecke der Fortpflanzung geschieht, scheint auch heute noch eine Sünde für ihn darzustellen. Die sich hier trotz einer auf der kognitiven Ebene erfolgten Loslösung von kirchlichen Konzepten manifestierende Verhaftung in katholischen Glaubensvorstellungen führt zu einer Übernahme der Deutung des Täters. Demnach rationalisiert der Betroffene durch den Gebrauch einer religiösen Semantik den erlebten sexuellen Missbrauch.

\section{Zusammenfassung und Fazit}

In den meisten rekonstruierten Umgangsformen der Interviewten mit dem sexuellen Missbrauch zeigte sich eine mehr oder weniger deutlich ausgeprägte Orientierung an katholischen Interpretationsvorlagen. Somit beeinflussen bis heute religiöse Glaubensvorstellungen sowie teilweise auch die Zugehörigkeit zur kirchlichen Gemeinde ihr retrospektives Erleben und Deuten der Viktimisierungserfahrungen. Auffällig ist hierbei, dass den Betroffenen die katholische Religion als Interpretationshilfe dient, sie also in ihrer Deutung der Geschehnisse auf die von der Kirche zur Verfügung gestellten geistigen Mittel zurückgreifen - und das obwohl der sexuelle Missbrauch in ihrem institutionellen Kontext und zuweilen sogar unter dem Deckmantel katholischer Glaubensvorstellungen stattfand. Selbst diejenigen Befragten, die aufgrund ihrer Erfahrungen versuchen, einen Bruch mit der Kirche zu vollziehen und beispielsweise aus dieser ausgetreten sind, bleiben in ihren Narrativen häufig in katholischen Semantiken verhaftet. Dies weist nicht nur auf ihr bis heute bestehendes ambivalentes Verhältnis zur Kirche - das Pendeln zwischen einer kritischen Distanz und einer emotionalen Verbundenheit ihr gegenüber - hin. Es veranschaulicht ebenso, wie schwer eine Loslösung von den institutionell vermittelten Glaubenskonzepten für Personen mit einer stark religiös geprägten Sozialisation ist.

Insofern greifen die Interviewten in der Auseinandersetzung mit der von ihnen erlebten sexuellen Gewalt nicht selten auf kollektive religiöse Interpretationsvorlagen zurück. Es kommt also weniger zur Entwicklung von individualisierten Deutungsmustern, in denen sich die Betroffenen von den im Rahmen ihrer Erziehung und Sozialisation verinnerlichten katholischen Überzeugungen emanzipieren. Dennoch lassen sich innerhalb der religiös geprägten Deutungen verschiedene Strategien des Umgangs mit dem Erlebten ausmachen. Diese hängen mit unterschiedlichen Formen 
der individuellen Religiosität zusammen. Zwar sind alle Befragten religiös sozialisiert und geprägt, jedoch bildeten sich, auch im Kontext individueller biografischer Konstellationen, unterschiedliche Formen von Religiosität aus, die einen Einfluss auf die subjektive Deutung des Missbrauchsgeschehens sowie den allgemeinen Umgang mit biografischen (Konflikt-) Erfahrungen haben. Diesen Überlegungen wird an anderer Stelle nachgegangen. ${ }^{8}$

Überdies wurde anhand der verschiedenen Interviewauszüge sichtbar, dass der häufig anzutreffende Rückgriff auf religiöse Interpretationsschemata eine ambivalente Wirkung auf die Verarbeitung der erlebten sexuellen Übergriffe entfaltet: Insbesondere die exemplarisch unter dem Deutungsmuster der religiösen Umbewertung angeführten Interviewsequenzen veranschaulichen, wie religiöse Überzeugungen innerhalb der intrapsychischen Emotionsregulation als Bewältigungsressource von traumatischen Erfahrungen dienen können (Grom, 2007). Hierauf verweist auch Haslbeck (2007), die in ihrer qualitativen Studie zu sexuellem Missbrauch und Religiosität herausarbeitet, dass religiöse Sinndeutungen den Heilungsprozess infolge derartiger Viktimisierungserfahrungen unterstützen können. So werden durch die nachträgliche Sinnverleihung und Sakralisierung des sexuellen Missbrauchsgeschehens, die sich im oben zitierten Deutungsmuster abzeichnet, Gefühle der Hilflosigkeit und Ohnmacht, des Schmerzes und der Verletztheit kompensiert. Mit der Verlagerung der Erfüllung von Erwartungen und Wünschen in eine transzendente Sphäre (wie hier einer gerechten Strafe für den Täter) findet eine Verminderung von $\mathrm{Ge}$ fühlen der Ungerechtigkeit und Wut statt. Allerdings geht mit der nachträglichen Sinnverleihung und Transzendierung tendenziell zugleich eine Unterdrückung der durch den Missbrauch ausgelösten Gefühle einher, da diese im diesseitigen Leben nicht zugelassen werden. Vielmehr tröstet die Vorstellung einer im Jenseits hergestellten Gerechtigkeit durch Gott über schmerzvolle Affekte und Wutgefühle hinweg, die so der Reflexion entzogen werden. Dies kann dazu beitragen, dass Handlungskonsequenzen im gegenwärtigen Leben ausbleiben - sei es eine Hilfesuche im Sinne einer therapeutischen Unterstützung zur Auf- und Verarbeitung der mit den traumatischen Erfahrungen verbundenen Gefühle oder aber eine Aufdeckung des Erlebten im sozialen Umfeld und eine Anzeige des Täters zu seiner strafrechtlichen Verfolgung.

8 Eine Arbeit, die sich ausführlicher mit jenem Themenkomplex der biografischen Bedeutung von Religiosität befasst, ist in Vorbereitung (Fernau, in Vorbereitung). 
In anderen Zusammenhängen scheint die katholisch geprägte Religiosität Prozesse der Aufarbeitung und Bewältigung des sexuellen Missbrauchs eindeutiger $\mathrm{zu}$ erschweren. Diesbezüglich wurde vor allem erkennbar, dass gerade die durch die religiöse Sozialisation mitbedingte starke emotionale Verbundenheit und Loyalität zur örtlichen Kirchengemeinde einigen Betroffenen eine Verarbeitung der sexuellen Gewalterfahrungen im Zuge einer Aufdeckung und aktiven Hilfesuche im sozialen und kirchlichen Umfeld erschwert. Auffällig ist dabei besonders, dass die religiöse Gemeinschaft nicht als stärkend und (be-)schützend erlebt bzw. gedeutet wird; vielmehr besteht tendenziell die Befürchtung, bei einer Offenbarung des Erlebten auf Ablehnung zu stoßen und mit negativen Fremdzuschreibungen konfrontiert zu werden. Die Bereitschaft zur Offenbarung der Viktimisierungserfahrungen wird also durch die Zugehörigkeit und Einbindung in die Kirchengemeinde nicht gefördert, sondern eher gehemmt. Darüber hinaus zeigte sich exemplarisch in einem Interviewauszug, wie die Verhaftung in katholischen Glaubensvorstellungen zu einer Übernahme der religiösen Neutralisierungstechniken des Geistlichen führen kann und somit eine vollständige Abkehr von den vom Täter herangezogenen Deutungsweisen verhindert. Auf diese Weise schreibt sich der Betroffene durch die Selbstinterpretation im Rahmen einer katholischen Semantik bis heute selbst die Schuld für den erfahrenen Missbrauch zu.

\section{Literatur}

Bange, D. (2007). Sexueller Missbrauch an Jungen: Die Mauer des Schweigens. Göttingen: Hogrefe.

Benkert, M. \& Doyle, T. P. (2009). Clericalism, religious duress and its psychological impact on victims of clergy sexual abuse. Pastoral Psychology, 58, 223-238.

Berliner, L. \& Conte, J. R. (1990). The process of victimization: The victim's perspective. Child Abuse and Neglect, 14, 825-837.

Bibel (1985). Altes und Neues Testament. Lutherbibel Standardausgabe. Stuttgart: Deutsche Bibelgesellschaft Stuttgart.

Bottoms, B. L., Nielsen, M., Murray, R. \& Filipas, H. (2003). Religion-related child physical abuse: Characteristics and psychological outcomes. Journal of Aggression, Maltreatment and Trauma, 8, 87-114.

Breuer, M. (2012). Religiöser Wandel als Säkularisierungsfolge. Differenzierungs- und Individualisierungsdiskurse im Katholizismus. Wiesbaden: VS.

Bullens, R. (1995). Der Grooming-Prozess - oder das Planen des Missbrauchs. In B. Marquardt-Mau (Hrsg.), Schulische Prävention gegen sexuelle Kindesmisshand- 
lung: Grundlagen, Rahmenbedingungen, Bausteine und Modelle (S. 55-67). Weinheim: Beltz.

Busch, H.-J. (2007). Religiosität aus soziologischer und sozialpsychologischer Perspektive. Psychoanalyse: Texte zur Sozialforschung, 11, 141-156.

Carr, A., Dooley, B., Fitzpatrick, M., Flanagan, E., Flanagan-Howard, R., Tierney, K., ... \& Egan, J. (2010). Adult adjustment of survivors of institutional child abuse in Ireland. Child Abuse and Neglect, 34, 477-489.

Crisma, M., Bascelli, E., Paci, D. \& Romito, P. (2004). Adolescents who experienced sexual abuse: Fears, needs and impediments to disclosure. Child Abuse and Neglect, $28,1035-1048$.

Doyle, T. P. (2003). Roman Catholic clericalism, religious duress, and clergy sexual abuse. Pastoral Psychology, 51, 189-231.

Doyle, T. P. (2006). Clericalism: Enabler of clergy sexual abuse. Pastoral Psychology, 54, 189-213.

Doyle, T. P. (2009). The spiritual trauma experienced by victims of sexual abuse by Catholic clergy. Pastoral Psychology, 58, 239-260.

Durkheim, E. (1981 [1912]). Die elementaren Formen des religiösen Lebens. Frankfurt am Main: Suhrkamp.

Fernau, S. (2013). „Weil ich die Widmung habe, alles als von Gott zu betrachten“ Zur religiösen Deutung sexueller Missbrauchserfahrungen im kirchlichen Kontext. Psychoanalyse - Texte zur Sozialforschung, 17, 17-30.

Fernau, S. (in Vorbereitung). Verstrickungen im Glauben - Zur biografischen Bedeutung von Religiosität vor dem Hintergrund sexuellen Missbrauchs durch katholische Geistliche.

Finkelhor, D. (1979). Sexually victimized children. New York, NY: Free Press.

Finkelhor, D. (1994). International epidemiology of child sexual abuse. Child Abuse and Neglect, 18, 409-417.

Freud, S. (1930). Das Unbehagen in der Kultur. Studienausgabe, Band IX. Frankfurt am Main: Fischer.

Grom, B. (2007). Religionspsychologie (3., vollständig überarbeitete Auflage). München: Kösel.

Haslbeck, B. (2007). Sexueller Missbrauch und Religiosität: Wenn Frauen das Schweigen brechen. Münster: LIT Verlag.

Haywood, T. W., Kravitz, H. M., Grossman, L. S., Wasyliw, O. E. \& Hardy, D. W. (1996). Psychological aspects of sexual functioning among cleric and noncleric alleged sex offenders. Child Abuse and Neglect, 20, 527-536.

Haywood, T. W., Kravitz, H. M., Wasyliw, O. E., Goldberg, J. \& Cavanaugh, J. L. (1996). Cycle of abuse and psychopathology in cleric and noncleric molesters of children and adolescents. Child Abuse and Neglect, 20, 1233-1243.

Helfferich, C. (2009). Die Qualität qualitativer Daten: Manual für die Durchführung qualitativer Interviews (3., überarbeitete Auflage). Wiesbaden: VS.

Herman, J. L. (1994). Die Narben der Gewalt: Traumatische Erfahrungen verstehen und überwinden. München: Kindler. 
John Jay College (2004). The nature and scope of sexual abuse of minors by Catholic priests and deacons in the United States, 1950-2002. Washington, DC: United States Conference of Catholic Bishops (USCCB).

Keupp, H., Straus, F., Mosser, P., Gmür, W. \& Hackenschmiedt, G. (2013). Sexueller Missbrauch, psychische und körperliche Gewalt im Internat der Benediktinerabtei Ettal. München: Institut für Praxisforschung und Projektberatung.

Knoblauch, H. (1999). Religionssoziologie. Berlin: De Gruyter.

Kruse, J. (2014). Qualitative Interviewforschung: Ein integrativer Ansatz. Weinheim: Beltz.

Laaser, M. (1991). Sexual addiction and clergy. Pastoral Psychology, 39, 213-235.

Lampe, A. (2002). The prevalence of childhood sexual abuse, physical abuse and emotional neglect in Europe. Psychosomatic Medicine and Psychotherapy, 48, 370-380.

Leygraf, N., König, A., Kröber, H.-L. \& Pfäfflin, F. (2012). Sexuelle Übergriffe durch katholische Geistliche in Deutschland. Eine Analyse forensischer Gutachten 20002010. Verfügbar unter http://www.dbk.de/fileadmin/redaktion/diverse_downloads/ Dossiers_2012/2012_Sex-Uebergriffe-durch-katholische-Geistliche_LeygrafStudie.pdf [12.12.2013]

London, K., Bruck, M., Cesi, S. J. \& Shuman, D. W. (2005). Disclosure of child sexual abuse: What does the research tell us about the ways that children tell? Psychology, Public Policy, and Law, 11, 194-226.

Lorenzer, A. (1981). Das Konzil der Buchhalter: Die Zerstörung der Sinnlichkeit.. Frankfurt am Main: Europäische Verlagsanstalt.

Lueger-Schuster, B. (2012). Psychotraumatologische Fragestellungen zu Gewalt und Missbrauch in der Katholischen Kirche. Wien: Universität Wien.

Luhmann, N. (1977). Funktion der Religion. Frankfurt am Main: Suhrkamp.

Luhmann, N. (1987). Die Unterscheidung Gottes. In N. Luhmann (Hrsgg.), Soziologische Aufklärung IV: Beiträge zur funktionalen Differenzierung der Gesellschaft (S. 236-353). Opladen: Westdeutscher Verlag.

Milanesi, G. (1976). Religionssoziologie: Wandlungsprozesse im religiösen Verhalten. Zürich: Benziger.

Mosser, P. (2009). Wege aus dem Dunkelfeld: Aufdeckung und Hilfesuche bei sexuellem Missbrauch an Jungen. Wiesbaden: VS.

Parsons, T. (1964). The social system (2. Auflage). New York, NY: Free Press.

Pickel, G. (2011). Religionssoziologie. Eine Einführung in zentrale Themenbereiche. Wiesbaden: VS.

Plante, T. G. (1996). Catholic priests who sexually abuse minors: Why do we hear so much yet know so little? Pastoral Psychology, 44, 305-310.

Plante, T. G. (2003). Priests behaving badly: What do we know about priest sex offenders? Sexual Addiction and Compulsivity, 9, 93-97.

Plante, T. G., Manuel, G. \& Bryant, C. (1996). Personality and cognitive functioning among hospitalized sexual offending Roman Catholic priests. Pastoral Psychology, $45,129-139$. 
Pollack, D. (1995). Was ist Religion? Probleme der Definition. Zeitschrift für Religionswissenschaft, 3, 163-190.

Pollack, D. (2001). Religion. In H. Joas (Hrsg.), Lehrbuch der Soziologie (S. 335-362). Frankfurt am Main: Campus.

Pollack, D. (2003). Säkularisierung - Ein moderner Mythos? Tübingen: Mohr Siebeck.

Przyborski, A. \& Wohlrab-Sahr, M. (2010). Qualitative Sozialforschung. Ein Arbeitsbuch. München: Oldenbourg.

Stark, R. \& Glock, C. Y. (1968). American piety: The nature of religious commitment. Berkeley, CA: University of California Press.

Stolz, J. (2008). Secularization theory and rational choice: An interpretation of macroand micro-theories of secularization using the example of Switzerland. In D. Pollack \& D. V. A. Olson (Eds.), The role of religion in modern societies (pp. 249270). London, UK: Routledge.

Storch, K. (2000). Kontingenzbewältigungen: Eine qualitative Untersuchung über den Zusammenhang von Konfessionalität bzw. Konfessionslosigkeit und der Bewältigung des kritischen Lebensereignisses „Wende“. In D. Pollack \& G. Pickel (Hrsg.), Religiöser und kirchlicher Wandel in Ostdeutschland 1989-1999 (S. 259-275). Opladen: Leske + Budrich.

Strauss, A. L. (1998). Grundlagen qualitativer Sozialforschung. Datenanalyse und Theoriebildung in der empirischen soziologischen Forschung (2. Auflage). München: Wilhelm Fink.

Strauss, A. L. \& Corbin, J. (1996). Grounded Theory. Grundlagen qualitativer Sozialforschung. Weinheim: Beltz.

Summit, R. C. (1983). The child sexual abuse accommodation syndrome. Child Abuse and Neglect, 7, 177-193.

Terry, K. J., Smith, M. L., Schuth, K., Kelly, J. R., Vollman, B. \& Massey, C. (2011). The causes and context of sexual abuse of minors by Catholic priests in the United States, 1950-2010. Washington, DC: United States Conference of Catholic Bishops (USCCB).

Wetzels, P. (1997). Gewalterfahrungen in der Kindheit: Sexueller Mißbrauch, körperliche Mißhandlung und deren langfristige Konsequenzen. Baden-Baden: Nomos.

Wohlrab-Sahr, M. (1995). Einleitung. In M. Wohlrab-Sahr (Hrsg.), Biographie und Religion: Zwischen Ritual und Selbstsuche (S. 9-26). Frankfurt am Main: Campus. 